\title{
El discurso sobre Dios en La carretera, de Cormac McCarthy
}

The Discourse on God in The Road, by Cormac McCarthy

José Antonio Millán Alba

Universidad Complutense de Madrid

Recibido: 10 diciembre de 2015

Aprobado: 20 diciembre de 2015

Pensamiento y Cultura | ISSN: 0123-0999 | eISSN: 2027-5331

pensam.cult | Vol. 17-2 | Diciembre de 2014 | pp. 182-207

DOI: 10.5294/pecu.2014.17.2.8 
La carretera es el relato de un viaje a pie emprendido por un padre y un hijo, que tratan de sobrevivir en una tierra devastada y cuyo objetivo es llegar al sur. Viaje por la carretera interestatal, en un mundo destruido, del que ha desaparecido toda vegetalidad y el mundo animal se reduce a la fugaz aparición de un perro cuya presencia se asimila a un recuerdo, o a una bandada de aves migratorias cuya única y fugaz presencia parece más bien resaltar su posterior y completa desaparición. Bosques y ciudades calcinados o que arden en unos fuegos postreros cuyo origen se desconoce, y cadáveres de seres humanos por doquier, en una casa, en un supermercado, empalados o acumulados en coches en posturas familiares como si les hubiera sorprendido una conflagración atómica o el estallido súbito de un volcán.

Por la carretera ya no circula nadie, pues apenas hay supervivientes; solo un camión en un fugaz momento, un grupo de personas apresados por otras, alguien que, abrasado, se sienta en ella para morirse, y un anciano que surge casualmente y luego continúa su camino. Pero la carretera sigue siendo el espacio de una travesía, la cual, en el inconsciente colectivo contemporáneo, fácilmente se asocia a los distintos viajes en los que se atraviesa, de norte a sur -o de este a oeste- la parte norte del continente americano, sus grandes espacios y sus horizontes sin fin, sus grandes obras de ingeniería, sus lagos, sus gigantescas quebradas y cañones.

Viajar. La literatura de los dos últimos siglos abunda en relatos de viajes, porque el viaje es movimiento, carencia de ataduras, libertad libre, ser, finalmente, otro, correspondencia y analogía de lo que se encuentra mientras dure ese tiempo de exultación abierto a cualquier expectativa, en el que lo imprevisto puede hacer en todo momento acto de presencia, en el que no hay nada estable sino la misma fugacidad, el movimiento embriagador del viaje, en el que pueden adoptarse, por último, todos los rostros, todas las máscaras, hacer momentáneamente nuestras todas las vidas. Hay en el viaje, o puede haberla, una excitación análoga a la que producen el sexo, el alcohol o los estupefacientes. Viaje de un marginal o de un vagabundo, viaje de quien vive, por decirlo así, a salto de mata, en cualquier caso de quien no posee nada. La narrativa contemporánea abunda, efectivamente, en relatos de viajes, la mayoría de las 
veces en busca de un origen -y en esto no se diferencia del viaje romántico-, o de una memoria y una identidad, personal o colectiva, todavía por encontrar. La carretera muestra, en cambio, un angustioso viaje de supervivencia en un mundo dominado por la muerte, pero participa de esa búsqueda de identidad, de ese espacio del otro con el que vivir que es propio de la narrativa posmoderna. Este viaje es, por otra parte, hacia el sur, un espacio mítico acuñado por las literaturas románticas y simbolistas, donde hace calor, la vida es fácil y florece por sí misma, y el aire y la luz están cargados de los colores, las refracciones y olores del mar, aunque el viaje de La carretera esté motivado por la imperiosa necesidad de encontrar calor en un mundo helado. Sin embargo, ese carácter mítico se redobla, porque, como en una epopeya prehistórica o futurista, o en un relato onírico, padre e hijo son los portadores del fuego, sin el cual no hay vida y por cuya significación tendremos que preguntarnos, lo que les confiere cierta dimensión sacral.

En el relato no hay marcas referenciales que permitan la localización del viaje; pueblos y ciudades pueden serlo de cualquier parte del mundo; los utensilios que se encuentran, así como los alimentos enlatados, o las bebidas que aparecen en bodegas y supermercados destruidos y saqueados no permiten una concreta localización. Solo he encontrado tres referencias, una de carácter histórico - una casa en el camino, con un porche de columnas dóricas en la que en el pasado los esclavos servían los alimentos en bandejas de plata, así como otra a campos de algodón-, otra lingüística, un objeto, ya en el sur, en el que hay algo escrito en inglés y en español, y otra, también en el sur -creo recordar que un sextante- fabricado en Cádiz o en Bristol, nueva referencia a las dos culturas. Este espacio resulta, así, a-tópico, pero parece inevitable situarlo en Estados Unidos, aunque solo sea por la presencia continua de la "interestatal", la carretera por la que marchan el padre y el hijo, que atraviesa el país de un extremo a otro.

Desde el punto de vista narrativo, el relato es isócrono, es decir, narra lo que ocurre en ese momento, en el momento en el que transcurre la acción, y está escrito en estilo directo. Apenas hay elementos reflexivos, ni monólogos, ni elementos de estilo indirecto. La narración avanza mediante alternancia de segmentos descriptivos y narrativos, que 
con mucha frecuencia se funden; esto es, el objeto de la narración está constituido por la descripción, cuyo punto de focalización es puramente objetivo, lo que ambos, padre e hijo, ven, sin que la narración remita -salvo en contadas ocasiones- a las respectivas vivencias subjetivas en relación con lo que miran. Esta fusión de segmentos puede ralentizar la acción, sobre todo si los componentes de la descripción son repetitivos, lo que es el caso, aunque el relato esté dividido en segmentos cortos y en la estructura gramatical de la frase apenas haya oraciones subordinadas, al igual que los conectores gramaticales tienden a desaparecer, lo que produce un efecto de brusquedad y de dureza de orden rítmico al que tampoco son ajenos los diálogos. Esta alternancia de relatos narrativos y descriptivos de carácter isócrono queda ocasionalmente interrumpida por analepsis y prolepsis que vehiculan los recuerdos, así como por relatos oníricos ("sueños tan llenos de color"', 2009, 21) que explicitan en el nivel simbólico el sentido del relato y anticipan la acción. Tienen mucha importancia en la construcción de la novela y en la comprensión y creación del sentido.

Pondré un ejemplo. El relato se inicia con un sueño, en el cual, dentro de una gruta, el niño conduce a su padre, "lo lleva de la mano"2 (ibídem, 9), al contrario de lo que ocurre en el resto de la narración. La luz de los dos "baila en la húmedas paredes de roca caliza"3 (ídem), sin que se sepa si lo que baila en las paredes es la luz procedente de algún tipo de lámpara que ambos llevan en la mano, o, más bien que ambos son luz (frente a la oscuridad del mundo exterior -"noches más tenebrosas que las tinieblas"4- (íd.), o la oscuridad de la gruta). Si ahora vamos al final de la novela, en el momento en el que el padre está a punto de morir, es el hijo el que se ocupa de él, como en el sueño inicial, y "a su alrededor todo era luz [...]. Cogió la taza y se alejó y cuando lo hizo la luz se movió con él"s (ib., 203).

\footnotetext{
"Dreams so rich in color" (McCarthy 2007, 21).

"Led him by the hand", ibídem, 3.

"Playing over the wet flowstone walls", ídem.

"Nights dark beyond darkness", ídem.

"There was light all about him [...]. He took the cup and moved away and when he moved the light moved with him", ibídem, 277.
} 
En el primer sueño, padre e hijo se encuentran "engullidos y extraviados en las entrañas de una bestia granítica. Humeros de piedra donde el agua goteaba y cantaba. Tañendo sin tregua en el silencio los minutos de la tierra y sus horas y días y años"6 (ib., 9). Momentos antes de morir el padre, se lee: "Goteo de agua [el agua aparece, así, como el elemento temporal por excelencia...]. Viejos sueños usurpando el mundo de la vigilia [el goteo era en el interior de la cueva, lo que remite directamente a la cueva del primer sueño...]. Huellas de ignotos animales en el limo gangrenado. Habían alcanzado en aquel frío pasadizo el punto sin retorno que únicamente se podía calcular desde el principio por la luz que llevaban consigo"' (ib., 205). El primer sueño se cumple, así, momentos antes de la muerte del padre y, en este sentido, resulta ser una figura del último, en el que se explicita el sentido de aquel. Los "ignotos animales en el limo gangrenado" que aparecen en el segmento de la muerte del padre, adquieren la figura en el relato del primer sueño en un ser monstruoso y abyecto que aparece en una "enorme estancia de piedra donde había un lago antiguo [nueva redundancia del agua y la temporalidad] y negro. Y en la orilla opuesta un ser que levantaba su chorreante boca del gour [obsérvese la triple redundancia en la descripción: lago, boca y gour, pues el gour es una boca o un cráter de agua similar a un lago] y miraba hacia la luz con unos ojos tan blancos y ciegos como los huevos de araña"8 (ib., 9).

Al principio, en el origen de la narración, como en la antigüedad del mundo, está lo abyecto, un animal ciego cuyos "huesos de alabastro" se funden con "las rocas que tenía detrás" (íd., asimilación anima-

6 "Swallowed up and lost among the inward parts of some granitic beast. Deep stone flutes where the water dripped and sang. Tolling in the silence the minutes of the earth and the hours and the days of it", ibídem, 3 .

7 "Drip of water [...]. Old dreams encroached upon the waking world [...]. Tracks of unknown creatures in the mortified loess. In that cold corridor they had reached the point of no return which was measured from the first solely by the light they carried with them", ibídem, 280.

8 "Great stone room where lay a black and ancient lake. And on the far shore a creature that raised its dripping mouth from the rimstone pool and stared into the light with eyes dead white and sightless as the eggs of spiders", ibídem, 3-4.

9 "Alabaster bones", "cast up in shadow on the rocks behind it", ibídem, 4. 
lidad-tierra). Presencia, pues, de una abyección en el fondo antiguo del mundo, o en el principio de cada relato, que es, a la vez, la materia misma de la tierra, su estructura ósea o rocosa, el "limo gangrenado". Esa presencia animal que habita en el fondo del tiempo, en el agua primigenia o en los años profundos, se conecta más adelante con el segundo ser humano con el que padre e hijo se encuentran: el primero, tan quemado como la ciudad que acaban de abandonar, se sienta en la carretera para morir. Aquel forma parte de un grupo que recorre la carretera en un camión diésel (tomemos la carretera ahora como una alegoría de la vida), asesinando y esclavizando, que les ataca y al que tienen que matar, era "el primer ser humano aparte del chico con quien había hablado en más de un año. Mi hermano a fin de cuentas"10 (ib., 59). Los ojos de araña del monstruo del sueño inicial, enteramente insensibles a la luz, se convierten ahora en "ojos fríos y movedizos", "de reptil" (la araña se ha metamorfoseado en reptil), que inevitablemente evocan el espectro simbólico de la serpiente y sus significaciones; y continúa el relato: "Mazacote de carne humana", cuyo lenguaje es la falsedad, el engaño, "que ha hecho de cada palabra del mundo una mentira"11 (ib., 60). Naturaleza humana y naturaleza cósmica se funden en la imagen del "limo gangrenado", que no deja de evocar a los hijos del limo del título de Octavio Paz - aunque la expresión proceda del poeta francés Gerardo de Nerval-, y resultan, así afectadas por un mismo mal originario que, para el hombre, desemboca en la destrucción por un lado de cualquier forma de verdad y en su completa animalización por otro y, para el cosmos, en el terrible paisaje apocalíptico de toda la obra, en devastación y esterilidad.

Por lo que atañe al mundo natural, este está iluminado por una luz gris plomo, luz cenicienta de un día gris, análoga a la ceniza que lo cubre todo, "polvo y ceniza por todas partes"12 (ib., 11). "Largos crepúsculos grises, los largos amaneceres grises"13 (ib., 12). El gris es el color domi-

10 "The first human being other than the boy that he'd spoken to in more than a year. My brother at last", ibídem, 75 .

11 "Reptilian", "cold and shifting eyes". "Claggy with human flesh", "who has made the world a lie every word", ídem.

12 "Dust and ash everywhere", ibídem, 7.

13 "Long gray dusks, the long gray dawns", ídem. 
nante es esta visión del mundo, tanto para la naturaleza como para las ciudades, resultado de la combustión de uno y otras: "Una casa incendiada en medio de un claro y más allá un tramo de pradera agreste y gris", "las formas de una ciudad destacaban en el gris general como un dibujo al carbón en medio del páramo"14 (ib., 12-13). La ausencia de color es una constante en este, en un mundo en el que padre e hijo buscan "algo que tuviera color"15 (ib., 10), se nos dice. Y ese gris no es el resultado de una saturación de colores ni del movimiento que anima al mundo natural, el cual también degenera en descomposición y muerte, en "limo gangrenado", como, por ejemplo, ocurre en el romanticismo negro o en algunos autores simbolistas, específicamente en Baudelaire, para quien la naturaleza está igualmente sometida al mal. El gris baudeleriano es un oxímoron cromático perfecto, la expresión cromática de una urdimbre, o de una textura aérea que sustenta y unifica las diversas y cambiantes modalidades de la luz (Baudelaire 2000, 1109):

Como quiera que los vapores de la estación -invierno o primavera-, leemos en el Salón de 1846, bañan, suavizan o disipan los contornos [lo que cuenta en la mirada de Baudelaire es la indefinición de los objetos, la impresión eternizada que producen], la naturaleza se parece a una peonza, la cual, movida por una velocidad acelerada, nos resulta gris, aunque contiene en si misma todos los colores ${ }^{16}$.

Nada de esto se produce aquí, pues la naturaleza descrita es una naturaleza estática, en gran medida producto de su devastación: "Se subió un poco más la mochila y observó el campo devastado", "en el pequeño valle la serpiente gris de un río. Inmóvil y precisa"17 (McCarthy

14 "A burned house in a clearing and beyond that a reach of meadowlands stark and gray", "the shape of a city stood in the grayness like a charcoal drawing sketched across the waste", ibídem, 8.

15 "Anything of color", ibídem, 4.

16 "Comme la vapeur de la saison, -hiver ou été- baigne, adoucit, ou engloutit les contours, la nature ressemble à un toton qui, mû par une vitessee accélérée, nous apparait gris, bien qu'il résume en lui toutes les couleurs" (Baudelaire 1991, 861).

17 "He shifted the pack higher on his shoulders and looked out over the wasted country", "in the little valley the still gray serpentine of a river" (McCarthy 2007, 6). 
$2009,11)$. A diferencia de lo que se ha visto en Baudelaire, la naturaleza aquí aparece sujeta a una inmovilidad fundamental. El mundo natural es un mundo de seres inertes, análogos a los restos fosilizados de vida humana ("restos fósiles en el fango reseco"18, ib., 15) o a los cadáveres expuestos en las casa y en lindes del campo, a los útiles inservibles de una civilización muerta. El otro término que me interesa aquí, en esta pequeña descripción, unido al de "inmóvil" es el de "preciso". En el discurso de esta obra, narración y descripción son, como ya he señalado, objetivas, de un realismo objetivo en el mismo sentido en el que se habla de la escritura de Flaubert, de tanta importancia para la contemporaneidad. La narración cuenta hechos desnudos, acciones mínimas y precisas que se suceden sin apenas modalización: "Cruzaron el río por un viejo puente de hormigón y varios kilómetros más adelante llegaron a una estación de servicio. Se quedaron observando desde la carretera. Creo que deberíamos ir a ver", "echar una ojeada. La naturaleza por la que vadearon se convertía en polvo a su paso"19 (ib., 11).

Este movimiento narrativo, que responde a la mirada que ve primero desde lejos y percibe el conjunto, para ver luego de cerca y enumerar, domina también en la descripción (íd.):

Se puso de pie y miró hacia el edificio. Los surtidores con sus mangueras todavía en su sitio. Las ventanas intactas. La puerta del taller estaba abierta y el hombre entró. Un armario metálico adosado a una pared. Registró los cajones [...]. Buenos manguitos de media pulgada. Un destornillador de trinquete. Miró a su alrededor. Un barril metálico lleno de basura. Entró en la oficina. Polvo y ceniza por todas partes. El chico permaneció en el umbral. Una mesa metálica, una caja registradora ${ }^{20}$.

18 "Fossil tracks in the dried sludge", ibídem, 12.

19 "They crossed the river by an old concrete bridge and a few miles on they came upon a roadside gas station. They stood in the road and studied it. I think we should check it out", "take a look. The weeds they forded fell to dust about them", ibídem, 6 .

20 "He stood and looked over the building. The pumps standing witht their hoses oddly still in place. The windows intact. The door to the service bay was open and he went in. A standing metal toolbox against one wall. He went through the drawers [...]. Good half-inch 
Narración y descripción están fundidas en una sucesión de fotogramas enumerativos tanto de acciones como de objetos, con lo que unas y otros tienden a igualarse.

En McCarthy, la percepción espacial opera primero fijándose en los conjuntos y enumerando luego sucesivamente los pormenores en su unidad, sin descomponerlos. No es desde luego la mirada de un miope, ni su imaginario tiende hacia un naturalismo de corte materialista en cuya descripción la mirada se gozase.

Junto al gris, procedente de la ausencia de luz y sol, de un camino iluminado por un "sol opaco moviéndose invisible más allá de las tinieblas" ${ }^{21}$ (ib., 17), el otro color que predomina en este paisaje es el negro, la ausencia completa de luz, que afecta tanto a la tierra "renegridas formas rocosas despuntando entre los bancos de ceniza y oleadas de ceniza elevándose para alejarse sobre la tierra baldía"22 (íd.), cuanto a la misma lluvia, la cual aparece iluminada por una "luz débil en derredor, temblorosa y sin origen, refractada en la lluvia de hollín a la deriva"23 (íd.). Una negrura que invade las noches y las hace ciegas e impenetrables.

También el agua es fundamentalmente negra; a veces gris. Los lagos son negros, el agua de los ríos es negra y gris, y gris es la nieve: "Está nevando, dijo el chico. Miró al cielo. Un solitario copo grisáceo que cayera de un tamiz. Lo atrapó en la palma de la mano y lo vio expirar como la postrera hostia de la cristiandad"24 (ib., 18). O en esta otra descripción: "Los húmedos copos grises bailando y cayendo hasta quedar en nada. Nieve fangosa y gris en las cunetas. Un agua negra corriendo por debajo de los empapados montones de ceniza"25 (íd.).

drive sockets. A ratchet. He stood looking around the garage. A metal barrel full of trash. He went into the office. Dust and ash everywhere. The boy stood in the door. A metal desk, a cashregister", ibídem, 6-7.

21 "The track of the dull sun moving unseen beyond the murk", ibídem, 14.

22 "Blackened shapes of rock standing out of the shoals of ash and billows of ash rising up and blowing downcountry through the waste", ídem.

23 "Faint light all about, quivering and sourceless, refracted in the rain of drifting soot", ibídem, 15.

24 "It's snowing, the boy said. He looked at the sky. A single gray flake sifting down. He caught it in his hand and watched it expire there like the last host of christendom", ibídem, 16.

25 "The wet gray flakes twisting and falling out of nothing. Gray slush by the roadside. Black 
Lo mismo ocurre con el agua congelada: "Acamparon en un banco de tierra al otro lado de un arroyo helado junto a la carretera. El viento había apartado la ceniza del hielo y el hielo estaba negro y el arroyo parecía un sendero de basalto que serpenteaba en el bosque"26 (ib., 32). Estos procesos de reduplicación de la materia mineral (agua transformada en basalto) son muy frecuentes en esta obra, en la que la naturaleza sufre un continuo proceso de mineralización, que convierte en estéril la materia orgánica. De hecho, es el mecanismo principal de esterilización del mundo natural. Un paisaje petrificado es un paisaje muerto. Incluso el agua de una cascada se vuelve oscura y gris: "El chico se adelantó, se puso en cuclillas y tocó el agua oscura con la mano [...]. La cascada casi caía en el centro de la poza. Una espuma gris giraba sobre si misma"27 (ib., 34). En sus orillas, "pequeños discos negros y fragmentos de cuarzo pulimentado"28 (íd.). Lo mismo ocurre en la percepción del mar, cuyo lecho se ha secado y por el que el padre va en un sueño "caminando por el lecho seco de un mar mineral agrietado y roto como un plato caído"29 (ib., 44).

Esta conjunción de agua y piedra, a lo que ha de unirse el asfalto de la carretera, domina el paisaje devastado de esta obra, del que está ausente toda forma de fertilidad orgánica. Es el mismo paisaje que puebla el universo imaginario de Baudelaire, de Piranesi, de Poe, de algunos momentos de Rimbaud, o de autores postsurrealistas como Pierre Jean Jouve o Pierre Emmanuel, con la diferencia de que aquí están ausentes los reflejos e iridiscencias de una luz artificial reflejada en aguas, charcos y cristales propios del primero. El mundo de La carretera es un mundo helado donde los haya. Frío y hielo, omnipresentes en la obra, son elementos isomorfos de la oscuridad y la negrura, del agua mineraliza y de la piedra. Y han de unirse a todas las representaciones de la muerte,

water running from under the sodden drifts of ash", ídem.

26 "They camped in a bench of land on the far side of a frozen roadside creek. The wind had blown the ash from the ice and the ice was black and the creek looked like a path of basalt winding through the woods", ibídem, 35-36.

27 "The boy walked out and squatted and laved up the dark water [...]. The waterfall fell into the pool almost at its center. A gray curd circled", ibídem, 38.

28 "Black disclets and bits of polished quartz", ídem.

29 "Trekking the dried floor of a mineral sea where it lay cracked and broken like a fallen plate", ibídem, 52 . 
el mal y el infierno a partir de la iconografía en torno al frío y la materia congelada, en la línea, por ejemplo, del Fausto de Thomas Mann.

En este gélido infierno de desolación en el que se ha convertido la tierra, donde el mundo hecho por el hombre ha quedado igualmente reducido a cenizas y escombros, de repente se abre camino una epifanía que recuerda al silencio de los espacios infinitos de Pascal (ib., 99-100):

Escarbaron en las ruinas de casas en las que antes no habrían entrado. Un cadáver flotando en el agua negra, [...] entre desperdicios y cañerías herrumbrosas [...]. Salió a la luz y se quedó allí de pie y fugazmente vio la verdad absoluta del mundo. El frío y despiadado girar de la tierra intestada. Oscuridad implacable [...]. El aplastante vacío negro del universo. $\mathrm{Y}$ en alguna parte dos animales perseguidos [¿figuras del padre y el hijo?] temblando como zorros escondidos en su madriguera. Tiempo prestado y mundo prestado y ojos prestados con que llorarlo ${ }^{30}$ [al llanto me referiré más adelante].

Incluso el mar participa de esta fría y oscura desolación cósmica (ib., 160):

Ahí estaba la playa gris y las olas encrespadas rompiendo opacas y plomizas [...]. Como la desolación de un mar extraño rompiendo en las playas de un mundo inaudito [...]. Más allá el vasto océano frío, meciéndose pesadamente como una [...] tina de lava", "y luego la línea de turbonada de ceniza gris [...]. Sentados con los talones hundidos en la arena vieron como el mar sombrío les lamía los pies. Frío, desolado, sin aves [...]. Miró al chico. Detectó la decepción en su cara. Siento que no sea azul, dijo. No pasa nada, dijo el chico ${ }^{31}$.

30 "They scrabbled through the charred ruins of houses they would not have entered before. A corpse floating in the black water, [...] among the trash and rusting ductwork [...]. He walked out in the gray light and stood and he saw for a brief moment the absolute truth of the world. The cold relentless circling of the intestate earth. Darkness implacable [...]. The crushing black vacuum of the universe. And somewhere two hunted animals trembling like groundfoxes in their cover. Borrowed time and borrowed world and borrowed eyes with which to sorrow it", ibídem, 130.

31 "Out there was the gray beach with the slow combers rolling dull and leaden [...]. Like the desolation of some alien sea breaking on the shores of a world unheard of [...]. Beyond that 
Otro de los elementos que construyen este universo imaginario es el viento, el cual desempeña un cometido de desestabilización de cualquier forma de vida que se pretenda firme: "Estaban atravesando la amplia llanura costera donde los vientos seculares los empujaban entre aullantes nubes de ceniza a buscar refugio donde pudieran", con "el cielo a mediodía negro como las bodegas del infierno", en una "tierra destripada y erosionada y árida. Huesos de seres muertos desparramados en los aguazales. Basurales de desperdicios anónimos"32 (ib., 132). El viento tiene, así la función de mostrar un mundo carente de basamento, de soporte, análoga a la omnipresencia de la muerte. Veámoslo en esta otra descripción, junto con varios de los componentes que ya se han señalado: "Se quedó escuchando el goteo del agua en el bosque. Lecho rocoso, este. El frío y el silencio. Las cenizas del mundo difunto trajinadas de acá para allá por los crudos y transitorios vientos en el vacío. Llevadas, esparcidas y llevadas de nuevo. Todo desencajado de su apuntalamiento. Sin soporte en el viento cinéreo"33 (ib., 14-15). Agente apocalíptico cuyo último cometido estriba en hacer patente la fragilidad, "la fragilidad de todo por fin revelada" 34 (ib., 27).

Como ya se ha podido ver, la tierra es un elemento devastado, estéril, seco y renegrido; tierra baldía, árida, silenciosa e infame, carente de toda fecundidad. Solo en tres ocasiones aparece algún alimento producido por esta tierra abrasada: unas colmenillas arrugadas, unas manzanas resecas y unas semillas viejas a punto de convertirse en polvo. Toda vegetación está muerta: "Un camino largo con hierba muerta.

the ocean vast and cold and shifting heavily like a [...] vat of slag", "and then the gray squall line of ash [...]. They sat with their heels dug into the sand and watched the bleak sea wash up at their feet. Cold. Desolate. Birdless [...]. He looked at the boy. He could see the disappointment in his face. I'm sorry it's not blue, he said. That's okay, said the boy", ibídem, 215. "They were crossing the broad coastal plain where the secular winds drove them in howling clouds of ash to find shelter where they could", "the noon sky black as the cellars of hell", "land [was] gullied and eroded and barren. The bones of dead creatures sprawled in the washes. Middens of anonymous trash", ibídem, 177.

33 "He lay listening to the water drip in the woods. Bedrock, this. The cold and the silence. The ashes of the late world carried on the bleak and temporal winds to and fro in the void. Carried forth and scattered and carried forth again. Everything uncoupled from its shoring. Unsupported in the ashen air", ibídem, 11.

34 "The frailty of everything revealed at last", ibídem, 28. 
Hiedra muerta a todo lo largo del muro, [...] árboles muertos al fondo [...]. Avanzó entre las ruinas de un viejo huerto de manzanos, negros tocones retorcidos, hierba muerta hasta las rodillas"35 (ib., 90-91). Los setos al borde de la carretera se convierten en zarzas negras y retorcidas, y las escasas tierras de labranza visibles todavía están "muertas hasta las raíces en los áridos terrenos de aluvión" ${ }^{36}$ (ib., 22).

De entre los componentes de esta tierra baldía asolada por el fuego, hay dos que destacan por su presencia continuada: la roca y la piedra de un lado, formaciones rocosas renegridas, lechos de piedra de los ríos, grutas, estancias de piedra, gargantas y simas erosionadas, la morfología de la roca es muy rica en este paisaje estéril, roca que lleva a veces a querer identificarse con ella: “ojalá mi corazón fuera de piedra" ${ }^{37}$ (ib., 15), con lo que la piedra adquiere una vidente a dimensión simbólica. Y del otro el bosque, pero un bosque fosilizado por la acción del fuego, árboles muertos que se derrumban y se convierten en ceniza o en troncos calcinados y desprovistos de ramas, o negros carbones en el agua, bosque fósil que ha de unirse a los procesos de petrificación señalados.

Por último el fuego, que resulta semánticamente ambiguo. Por un lado, aparece como el elemento de destrucción por excelencia, cuyas causas se desconocen. Pero, a la vez, es calor y es luz, es decir, origen y fuente de vida. Frente a la acción del frío, la suya es enteramente benéfica. En la medida en que es también luz, su presencia es capaz de producir algo de color en un mundo gris y helado: "Había incendios activos todavía arriba en las montañas y por las noches podían ver sus luces de un naranja intenso entre el hollín que descendía" ${ }^{38}$ (ib., 28). Su presencia, por último, se asocia a la recuperación de una memoria y una identidad que amenazan con perderse (ib., 29):

35 "A long drive with dead grass. Dead ivy along a stone wall, [...] dead trees beyond [...]. He made his way down through the ruins of an old apple orchard, black and gnarly stumps, dead grass to his knees", ibídem, 118.

36 "Dead to the root along the barren bottomlands", ibídem, 21.

37 "If only my heart were stone", ibídem, 11.

38 "There were fires still burning high in the mountains and at night they could see the light from them deep orange in the sootfall", ibídem, 30. 
Todo estaba encendido, como si el sol ausente hubiera vuelto por fin. La nieve naranja y temblorosa. Un incendio en el bosque se abría paso por los cerros de pura yesca, llameando y titilando como una aurora boreal contra el cielo nublado [...]. El color de todo aquello removía en él algo olvidado hacía tiempo. Haz una lista. Recita una letanía. Recuerda ${ }^{39}$.

Esa identidad personal está, efectivamente, vinculada a las listas, es decir, a la enumeración y desglose de cosas o de acciones. A ello está asociada la existencia del lenguaje (ib., 70):

Intentó pensar en algo que decir, pero no pudo. No era la primera vez que tenía esa sensación, más allá del entumecimiento y la sorda desesperación. Como si el mundo se encogiera en torno a un número de entidades desglosables. Las cosas cayendo en el olvido y con ellas sus nombres. Los colores [obsérvese la identificación nombres-colores]. Los nombres de los pájaros. Alimentos. Por último los nombres de cosas que uno creía verdaderas. Más frágiles de lo que él había pensado. ¿Cuánto de este mundo había desaparecido ya? El sagrado idioma desprovisto de sus referentes y por tanto de su realidad. Rebajado como algo que intenta preservar el calor ${ }^{40}$.

La garantía de la perdurabilidad del lenguaje, la condición de su realidad es algo exterior a él; se sitúa en la realidad del mundo. En la medida en que este desaparece, desaparece también el lenguaje, y con él la memoria. La realidad "se encoge", se empequeñece, y el

39 "Everything was alight. As if the lost sun were returning at last. The snow orange and quivering. A forest fire was making its way along the tinderbox ridges above them, flaring and shimmering against the overcast like the northern lights [...]. The color of it moved something in him long forgotten. Make a list. Recite a litany. Remember", ibídem, 31.

40 "He tried to think of something to say but he could not. Hed had this feeling before, beyond the numbness and the dull despair. The world shrinking down about a raw core of parsible entities. The names of things slowly following those things into oblivion. Colors. The names of birds. Things to eat. Finally the names of things one believed to be true. More fragile than he would have thought. How much was gone already? The sacred idiom shorn of its referents and so of its reality. Drawing down like something trying to preserve heat", ibídem, 88-89. 
lenguaje queda, así, "rebajado". Pues las palabras son tratadas como cosas. El individuo quiere entonces "decir", pero no "puede". El texto establece una relación directa entre realidad-palabras-color-calor es decir, expresión de vida.

Esas listas de nombres a las que está vinculada la memoria y que corren el riesgo de sumirse en el olvido en la misma medida que las cosas, revisten también la forma de un mapa o de un listín telefónico, que por un lado garantizan y por otro justifican la inserción de los individuos en el mundo (ib., 136):

El chico asintió con la cabeza y se quedó mirando el mapa. El hombre le observó [...]. Él mismo había mirado mapas de niño, poniendo el dedo sobre el pueblo donde vivía. Igual que buscaba el apellido de su familia en el listín de teléfonos. Ellos entre muchos otros. Cada cosa en su sitio. Justificados en el mundo ${ }^{41}$.

De la misma forma que las listas-lenguaje son condición de la memoria, esto es, del pasado, también lo son del futuro. En la medida en que estas desaparezcan, desaparecerá también la posibilidad de aquél. Entonces el devenir temporal se fija en un presente continuo, en un día, en una hora: "Sin listas de cosas que hacer. El día providencia de sí mismo. La hora. No hay después. El después es esto"42 (ib., 45). Y "esto", el mundo presente, es lo que se encuentra en la carretera.

Tampoco existe el pasado, porque lo pasado se identifica con "esto", y lo que no cabe es inventárselo, pues la ficción equivale aquí a falta de verdad: "A veces el niño le hacía preguntas acerca del mundo que para él no era ni siquiera un recuerdo. Se esforzaba mucho por responder. No existe pasado. ¿A ti qué te gustaría? Pero dejó de inventarse cosas

41 "The boy nodded. He sat looking at the map. The man watched him [...]. He'd pored over maps as a child, keeping one finger on the town where he lived. Just as he would look up his family in the phone directory. Themselves among others, everything in its place. Justified in the world", ibídem, 182.

42 "No lists of things to be done. The day providential to itself. The hour. There is no later. This is later", ibídem, 54 . 
porque esas cosas tampoco eran verdad y decirlas le hacía sentir mal"43 (ib., 45). El pasado es algo perdido en la misma medida en que el mundo también es algo perdido, en que ya no cabe pertenecer a él, en que la tierra, presa de un mal fundamental, ha dejado de ser un hogar, y se ha convertido en ceniza (ib., 116).

Se volvió y miró al chico. Quizá comprendía por primera vez que para el chico él también era un extraterrestre. Un ser de un planeta que ya no existía y cuyas historias eran sospechosas. No podía inventar para gusto del chico el mundo que había perdido sin inventar también dicha pérdida [...], él no podía avivar en el corazón del niño lo que en el suyo propio eran cenizas ${ }^{44}$.

Frente al relato de ficción están las cosas, las listas de nombrescosas, los objetos del mundo, técnicos y mecánicos. Su enumeración y desglose, como si realidad fuese un gigantesco catálogo o una interminable enciclopedia, el ideal enciclopédico del mundo propio del homo faber que triunfa en el XIX y en el que se inscriben el realismo y el naturalismo literarios. Llama la atención que en todo el relato apenas haya referencias de orden cultural, ni tan siquiera para mostrar el fracaso de una hipotética visión humanista del mundo. La que hay es profundamente despectiva, el recuerdo de una biblioteca: "Años después había estado en las ruinas calcinadas de una biblioteca donde los libros yacían renegridos en charcos de agua" ${ }^{45}$ (ib., 139), como si la sabiduría humana y sus promesas felicidad contenidas en los libros fuesen

43 "Sometimes the child would ask him questions about the world that for him was not even a memory. He thought hard how to answer. There is no past. What would you like? But he stopped making things up because those things were not true either and the telling made him feel bad", ibídem, 53-54.

44 "He turned and looked at the boy. Maybe he understood for the first time that to the boy he was himself an alien. A being from a planet that no longer existed. Tha tales of which were suspect. He could not construct for the child's pleasure the world he'd lost without constructing the loss as well [...], he could not enkindle in the heart of the child what was ashes in his own", ibídem, 153-154.

45 "Years later he'd stood in the charred ruins of a library where blackened books lay in pools of water", ibídem, 187. 
una mentira tan enfatuada como esos mismos libros hinchados por el agua: "Los estantes volcados. Rabia contra las mentiras dispuestas en millares de hileras sucesivas. Cogió unos de los libros y pasó las páginas tan hinchadas. Él no hubiera dado valor a la más mínima cosa basada en un mundo futuro" 46 (íd.).

Frente al relato de ficción -soñar con un mundo que nunca existió o que nunca existirá equivale a rendirse, a dejar de luchar, a evadirse $^{47}$ (ib., 141) - está también la historia. El relato de la pérdida de ese mundo no se nos cuenta. Solo se nos dice lo que pasaba al principio: "En aquellos primeros años las carreteras estaban pobladas por refugiados envueltos hasta arriba en sus harapos. Con mascarillas y gafas protectoras [...]. Sus carretillas repletas de deshechos. Tirando de carromatos o carritos de supermercado [...]. Hollejos de hombres sin credo tambaleándose por los pasos elevados como emigrantes en una tierra salvaje" 48 (ib., 27). La historia ha dado, sin embargo, avisos de ello; o al menos esta puede ser leída así: "Le había dado por ver un mensaje en cada ejemplo de la historia tardía, un mensaje y una advertencia, y eso resultó ser este retablo de muertos y devorados" 49 (ib., 71), es decir, el mundo presente, lo que antes se ha nombrado como "esto" o, en otros términos, lo que aparece en la carretera, donde "no hay interlocutores de Dios. Se han ido y me han dejado aquí solo y se han llevado consigo el mundo. Duda: ¿En qué difiere el nunca será de lo que nunca fue?" ${ }^{50}$ (ib., 30). Y "esto" que aparece en el presente es una repetición, algo que ya ha sido (ib., 70):

46 "Shelves tipped over. Some rage at the lies in their thousands row on row. He picked up one of the books and thumbed through the heavy bloated pages. He'd not have thought the value of the smallest thing predicated on a world to come", idem.

47 Ibídem, 189.

48 "In those first years the roads were peopled with refugees shrouded up in their clothing. Wearing masks and goggles [...]. Their barrows heaped with shoddy. Towing wagons or carts [...]. Creedless shells of men tottering down the causeways like migrants in a feverland", ibídem, 28.

49 "He'd come to see a message in each such late history, a message and a warning, and so this tableau of the slain and the devoured did prove to be", ibídem, 91.

50 "There are no godspoke men. They are gone and I am left and they have taken with them the world. Query: How does the never to be differ from what never was?”, ibídem, 32. 
Se detuvo y miró más allá de los campos. Viento en el este [no se sabe si la referencia al este tiene un valor simbólico, o no]. La blanda ceniza moviéndose en los surcos [...]. Él ya lo había visto antes. Dibujos de sangre seca en los rastrojos y grises vísceras enroscadas allá donde los muertos habían sido destripados como animales y llevados a rastras. Sobre el muro del fondo un friso de cabezas humanas, todas de parecido rostro, resecas y hundidas con la sonrisa rígida y los ojos marchitos ${ }^{51}$.

La imagen que se nos describe a continuación es la de un friso, o un "retablo", como se nos ha dicho antes, en el que estuviese representada toda la violencia humana, todo el horror acumulado en la historia, historia a la vez ancestral y presente, intemporal y cotidiana, que adquiere la formulación de un grafiti actual o el montaje de un plano cinematográfico (ib., 71):

[Las cabezas] tenían aros de oro en sus coriáceas orejas y el viento hacía bailar sus escasos y raídos cabellos. Los dientes como empastes en sus alvéolos, los toscos tatuajes grabados con alguna tintura de elaboración casera descoloridos a la pauperizada luz del sol. Arañas, espadas, dianas. Un dragón, consignas rúnicas, credos mal escritos. Viejas cicatrices con motivos viejos pespunteados en sus bordes. Las cabezas no deformadas a porrazos habían sido desolladas y los nuevos cráneos pintados y rubricados de parte a parte de la frente a garabatos y una de aquellas calaveras peladas tenía las suturas cuidadosamente entintadas como en un plano para montaje ${ }^{52}$.

51 "He stopped and looked across the fields. Wind in the east. The soft ash moving in the furrows [...]. He'd seen it all before. Shapes of dried blood in the stubble grass and gray coils of viscera where the slain had been field-dressed and hauled away. The wall beyond held a frieze of human heads, all faced alike, dried and caved with their taut grins and shrunken eyes", ibídem, 90 .

52 "They wore gold rings in their leather ears and in the wind their sparse and ratty hair twisted about on their skulls. The teeth in their sockets like dental molds, the crude tattoos etched in some homebrewed woad faded in the beggared sunlight. Spiders, swords, targets. A dragon. Runic slogans, creeds misspelled. Old scars with motifs stitched along their borders. The heads not truncheoned shapeless had been flayed of their skins and the raw 
El friso sirve como introducción para lo que va a venir después. "Hay gente en la carretera" (íd.). Podrían ser los buenos, pregunta en algún momento el chico. No. Como ya sabemos, es gente que no dialoga con Dios. El mundo está dividido en buenos y malos. “¿Querías conocer a los malos?", le pregunta el padre. "Pues ya los has visto”. Y el mal produce miedo, miedo, como todos los miedos a un mal futuro, a un daño por venir que a la vez es actual y pasado y que nos debilita. Su contrario es la fe, la confianza, que nos asegura. Es la fe que el chico tiene respecto de su padre ("Yo siempre te creo. / Me parece que no. / Claro que sí. Tengo que creerte" 53 , ib., 138). Pero para que la fe sea posible resulta del todo necesario la existencia de un bien y un mal, de una verdad y una mentira. El mal radica aquí en comerse a sus semejantes, en someterlos y devorarlos, incluido el hecho de abortar para comerse al feto, un bebé destripado y decapitado asándose en un espeto, es decir, en incorporárselos, con todas las reminiscencias antropológicas y teológicas que ello implica. Los buenos son, por el contrario, los que han tomado la decisión radical, la determinación de no hacerlo nunca, en ninguna circunstancia, a lo que responde el diálogo entre el padre y el hijo sobre esta cuestión (ib., 98):

Se volvió y le miró. Parecía que hubiera estado llorando. / Habla. / Nosotros nunca nos comeríamos a nadie, ¿verdad? / No. Claro que no. / ¿Aunque estuviéramos muriéndonos de hambre? / [...] No. No lo haríamos. / Pase lo que pase. / Pase lo que pase. / Porque nosotros somos de los buenos. / Sí. / Y llevamos el fuego. / Y llevamos el fuego. Así es. / Vale ${ }^{54}$.

Si hay algo sobre lo que habla la literatura contemporánea, si hay en ella algún tema continuamente presente, es el mal, con sus distintas

skulls painted and signed across the forehead in a scrawl and one white bone skull had the plate sutures etched carefully in ink like a blueprint for assembly", ibídem, 90.

53 "I always believe you. / I don't think so. / Yes I do. I have to", ibídem, 185.

54 "He turned and looked. He looked like he'd been crying. / Just tell me. / We wouldn't ever eat anybody, would we? / No. Of course not. / Even if we were starving? / [...] No. We wouldn't. / No matter what. / No. No matter what. / Because we're the good guys. / Yes. / And we're carrying the fire. / And we're carrying the fire. Yes. / Okay", ibídem, 128-129. 
morfologías, sus consecuencias históricas y cotidianas, la omnipresencia del dolor infligido al otro, la impotencia y la muerte. Esto es especialmente visible hoy. Y ello hasta el punto de que no cabe entender el arte contemporáneo sin tener como correlato esa presencia del mal, que amenaza, cuando no gangrena toda forma de vida verdaderamente humana.

A esa amenaza, o a ese miedo, que tiene bastante más importancia en nuestras vidas de lo que puede parecer, que engendra pereza, parálisis, anquilosamiento y muerte es a lo que sucumbe la madre, en un proceso paulatino de autodestrucción. En la escena que se narra de nuevo aparece el fuego, un fuego cotidiano con toda su ambigüedad, $y$ que se concreta en la llama de un cigarro (ib., 47-48):

No puedes protegernos. Dices que darías la vida por nosotros pero $\dot{i d e}$ qué sirve eso? [...]. Tarde o temprano nos cazarán y nos matarán. A mí me violarán. A él también. Nos van a violar y después de matarnos nos devorarán pero tú no quieres reconocerlo. Tú prefieres esperar a que eso pase. Pues yo no. No puedo. Se quedó allí sentada fumando un tallo enclenque de parra seca como si fuera una especie de extraño cigarro puro [...]. Ella le miró del otro lado de la pequeña llama. Antes hablábamos de la muerte, dijo. Ya no. ¿Y sabes por qué? / No. No lo sé. / Porque la muerte está aquí. No hay otra cosa de que hablar [...]. Por lo que a mí respecta mi única esperanza es la nada eterna y la deseo con toda mi alma ${ }^{55}$.

Frente a todo ello, hay una orden, una orden que, en oposición a la muerte, es vida, una orden que se presenta como absoluto porque procede de Dios. Vinculada a ella, la noción del deber como respuesta a esa orden que está, a su vez, asociada a la bondad: "Querías saber qué

55 "You can't protect us. You say you would die for us but what good is that? [...] Sooner or later they will catch us and they will kill us. They will rape me. They'll rape him. They are going to rape us and kill us and eat us and you won't face it. You'd rather wait for it to happen. But I can't. I can't. She sat there smoking a slender lenght of dried grapevine as if it were some rare cheroot [...]. She watched across the small flame. We used to talk about death, she said. We don't any more. Why is that? / I don't know. / It's because it's here. There's nothing left to talk about [...]. As for me my only hope is for eternal nothingness and I hope it with all my heart", ibídem, 56-57. 
pinta tenían los malos. Pues ya lo sabes [...]. Mi deber es cuidar de ti. Dios me asignó esa tarea [...]. ¿Lo entiendes? [...]. Se quedó allí sentado con la manta por capucha. Al cabo de un rato levantó la vista. ¿Todavía somos los buenos?, dijo. / Sí. Todavía somos los buenos"56 (ib., 61).

La presencia de los malos está focalizada en torno al color rojo, "vestidos con prendas de lo más variado, todos ellos con bufandas rojas alrededor del cuello. Rojas o naranjas, lo más parecido al rojo que pudieron encontrar" ${ }^{57}$ (ib., 71). Un desfile o una marcha militar como en las películas futuristas del tipo Mad Max, en la que los movimientos resultan puramente mecánicos: "Un ejército con zapatillas deportivas, pisando fuerte. Portando trozos de tubería de tres palmos de largo envueltos en cuero [...]. A algunos de los tubos les habían ensartado tramos de cadena provistos en su extremo de cachiporras de toda clase. Pasaron de largo en ruidoso desfile, balanceándose como juguetes de cuerda" ${ }^{58}$ (íd.). Detrás de esta marcha triunfal, el cortejo que les acompaña en una nueva forma de abyección: "La falange que los seguía portaba una especie de lanzas adornadas con cintas y borlas [...]. Detrás de ellos carros tirados por esclavos con arneses y repletos de mercancía de guerra y más atrás las mujeres, como una docena, algunas de ellas embarazadas, y por último un conjunto adicional de catamitas mal vestidos para el frío y provistos de dogales y enyuntados entre si'”59 (ib., 71-72). Poco más adelante, padre e hijo llegan a una elegante casa con amplio

56 "You wanted to know what the bad guys look like. Now you know [...]. My job is to take care of you. I was appointed to do that by God [...]. Do you understand? [...] He sat there cowled in the blanket. After a while he looked up. Are we still the good guys? he said. / Yes. We're still the good guys", ibídem, 77.

57 "Dressed in clothing of every description, all wearing red scarves at their necks. Red or orange, as close to red as they could find", ibídem, 91.

58 "An army in tennis shoes, tramping. Carrying three-foot lengths of pipe with leather wrappings [...]. Some of the pipes were threaded through with lenghts of chain fitted at their ends with every manner of bludgeon. They clanked past, marching with a swaying gait like wind-up toys", ídem.

59 "The phalanx following carried spears or lances tasseled with ribbons [...]. Behind them came wagons drawn by slaves in harness and piled with good of war and after that the women, perhaps a dozen in number, some of them pregnant, and lastly a suppementary consort of catamites illclothed against the cold and fitted in dogcollars and yoked each to each", ibídem, 91-92. 
porche de columnas dóricas. El chico se niega a entrar, pero el padre le obliga: tienen que encontrar algo de comer. El chico llora. Dentro, el padre abre una trampilla cerrada con llave (ib., 86-87):

Acurrucados junto a la pared del fondo había hombres y mujeres desnudos, todos tratando de ocultarse, protegiéndose el rostro con las manos. En el colchón yacía un hombre al que le faltaban las dos piernas hasta la cadera, los muñones quemados y ennegrecidos. El olor era insoportable [...]. Ayúdenos, dijeron en voz baja. Por favor, ayúdenos [...]. Cuatro barbudos y dos mujeres [integrantes del desfile anterior] venían hacia la casa atravesando el campo ${ }^{60}$.

Más adelante, de nuevo en la carretera, encuentran a un viajero al que dan alcance. Resulta ser un viejo y, en este sentido, voz de un mundo inmediatamente anterior, aquel que ha conducido a la devastación actual, un antiguo mendigo, menudo y encorvado, de olor nauseabundo. A petición del chico le dan alimentos y pasan la noche con él al calor de una hoguera. A un comentario sobre Dios por parte del padre, el viejo contesta rotundamente: "Dios no existe. Y nosotros somos sus profetas" ${ }^{\prime 1}$ (ib., 127). Con lo que de nuevo la palabra humana aparece como una fundamental mentira. En el momento de despedirse, el padre le dice que le han ayudado a instancias del chico, y añade la razón de esa ayuda: "Quizá el chico cree en Dios". "Lo superará", contesta el viejo. "No", dice el padre, "no lo superará"62 (íd.).

En oposición a "esto", con todas sus significaciones, atravesado por la carrera, está el amor humano. En este sentido, La carretera puede leerse como un relato alegórico de la ausencia y la presencia del amor humano, vinculado al amor de Dios y a su capacidad creadora: "Recordaba

60 "Huddled against the back wall were naked people, male and female, all trying to hide, shielding their faces with their hands. On the mattress lay a man with his legs gone to the hip and the stumps of them blackened and burnt. The smell was hideous [...]. Help us, they whispered. Please help us [...]. Coming across the field toward the house were four bearded men and two women", ibídem, 110-111.

61 "There is no God and we are his prophets", ibídem, 170.

62 "Maybe he believes in God". "He'll get over it". "No he won't", ibídem, 174. 
haberse despertado una noche [...]. Las ascuas todavía vivas de una lumbre [...]. Se levantó y caminó un trecho y descalzo en la arena vio aparecer las pálidas crestas de las olas [estamos, así en el sur...]. Cuando volvió al fuego se arrodilló junto a ella y acarició sus cabellos mientras dormía y dijo que si él fuera Dios habría creado el mundo tal cual sin ninguna diferencia" ${ }^{63}$ (ib., 163). Amor ciertamente físico, del que hay unas mínimas pinceladas en algunas analepsis relativas a la presencia feliz de la esposa, y amor que va más allá. Por un parte está vinculado a la fecundidad, la luz, el calor y el canto ("algunas noches despertaba en medio del negro páramo helado saliendo de mundos de amor humano suavemente coloreados, cantos de pájaros, el sol"64, ib., 200). Y por otro a la bondad, que en el texto es ayuda, capacidad de acogida, acción de gracias, alegría. En el momento de la muerte del padre, cuando el chico va aquedarse solo, y acordándose de un niño que entrevieron por el camino, este le pregunta a su padre: "Pero, ¿quién lo encontrará si es que se ha perdido? ¿Quién encontrará al niño? / La bondad encontrará al niño Así ha sido siempre y así volverá a ser”65 (ib., 206).

Bondad, vida y aliento que proceden de Dios, en ese sur por fin alcanzado, el encuentro con otros que también son portadores del fuego en el corazón (ib., 209-210):

La mujer al verle lo rodeó con sus brazos y lo estrechó. Oh, dijo, me alegro tanto de verte. A veces le hablaba de Dios. Él intentó hablar con Dios pero lo mejor era hablar con su padre y eso fue lo que hizo y no se le olvidó. La mujer dijo que eso estaba bien. Dijo que el aliento de Dios era también el de él aunque pasara de hombre a hombre por los siglos de los siglos ${ }^{66}$.

63 "He remembered waking once on such a night [...]. Faint deep coals of the driftwood fire [...]. He rose and walked out and stood barefoot in the sand and watched the pale surf appear [...]. When he went back to the fire he knelt and smoothed her hair as she slept and he said if he were God he would have made the world just so and no different", ibídem, 219.

64 "In the nights sometimes now he'd wake in the black and freezing waste out of softly colored worlds of human love, the songs of birds, the sun", ibídem, 272.

65 "But who will find him if he's lost? Who will find the little boy? / Goodness will find the little boy. It always has. It will again", ibídem, 281.

66 "The woman when she saw him put her arms around him and held him. Oh, she said, I am 
¿A qué apunta, finalmente, el componente temático del fuego? Algunas de sus significaciones se han señalado ya. Pero conviene recordar el comienzo de la agonía del padre, precisamente porque el "fuego del corazón”, el que el chico lleva dentro, en ese momento se concreta en luz, "a su alrededor todo era luz" ${ }^{67}$ (ib., 203). El final del segmento establece la equivalencia entre la luz y la palabra, su fusión: "Se quedó acostado mirando al chico junto al fuego. Quería ser capaz de ver. Mira todo esto, dijo. No hay un solo profeta en la larga crónica de la Tierra que no encuentre hoy aquí su razón de ser. Teníais razón, hablarais de lo que hablarais"68 (ib., 203). Pero esa palabra-luz no es una palabra cualquiera; es palabra pronunciada por Dios, y esa palabra es el chico mismo, "lo único que había entre él [el padre] y la muerte"69 (ib., 27): "Si él [el niño] no es la palabra de Dios, Dios no ha hablado nunca"70 (ib., 10). La palabra, y palabra de Dios, se presenta, así, como la significación última del fuego, lo único capaz de detener la devastación y la muerte, la vida que brota de Dios, luz que radica en el "corazón” y que finalmente permite por fin "ser capaz de ver". No cabe, por tanto, extrañarse, de que en determinados momentos el chico revista un carácter sagrado ("se sentó a su lado y acarició sus pálidos cabellos enmarañados. Cáliz de oro, bueno para albergar a un dios [...]. Todo ello como en un antiguo ungimiento"71, ib., 58-59). Ni tampoco de que la palabra, el “idioma sagrado”, deje de ser la enumeración de un catálogo y pueda por fin convertirse en narración: “Que así sea. Evoca las formas. Cuando no tengas nada más inventa ceremonias e infúndeles vida"72 (ib., 59).

so glad to see you. She would talk to him sometimes about God. He tried to talk to God but the best thing was to talk to his father and he did talk to him and he didn't forget. The woman said that was all right. She said the breath of God was his breath yet though it pass from man to man through all of time", ibídem, 286.

67 “There was light all about him", ibídem, 277.

68 "He lay watching the fire. He wanted to be able to see. Look around you, he said. There is no prophet in the earth's long chronicle who's not honored here today. Whatever form you spoke of you were right", ídem.

69 "All that stood between him and death", ibídem, 29.

70 "If he is not the word of God God never spoke", ibídem, 5.

71 "He sat beside him and stroked his pale and tangled hair. Golden chalice, good to house a god [...]. All of this like some ancient anointing", ibídem, 74-75.

72 "So be it. Evoke the forms. Where you've nothing else construct ceremonies out of the air and breathe upon them", ibídem, 74 . 
Quiero, por último, señalar un aspecto al que ya he aludido pero que he ido dejando deliberadamente de lado. Me refiero al llanto. Hay dos tipos de llanto en el texto que se dan predominantemente en el chico: uno producido por el miedo, y otro producido por la compasión. El primero tiene una concreta referencia a la persona que sufre ese miedo, y librarnos de él, o de la causa que lo origina, es un bien para cada uno; pero en el segundo no hay esa referencia: se tiene compasión por la desgracia ajena sencillamente porque es una desgracia, sin que ello se refiera a quien tiene ese sentimiento; en él se mira directamente al otro, sin que nos miremos de reojo a nosotros mismos: es este tipo de llanto el que predomina en el texto, como lo propio del chico. Pero hay un tercero, muy emparentado con este, pues, aunque "todas las cosas bellas y armónicas que uno conserva en su corazón tienen una procedencia común en el dolor"73 (ib., 45) -dolor que aquí radica en "el hecho de nacer en la aflicción y la ceniza"74 (íd.)-, y es el que siente el padre viendo dormir al chico; entonces "había momentos en los que empezaba a sollozar sin poder controlarse pero no por la idea de la muerte. No estaba seguro de cuál era el motivo pero pensaba que tenía que ver con la belleza o con la bondad"75 (ib., 99). Bondad y Belleza pueden, efectivamente, conmovernos hasta lo más íntimo, pero para ello es primero preciso que brillen con todo el fuego de lo valioso, y luego que su voz (de nuevo la conjunción fuego-luz-palabra) resuene en nosotros, para lo cual es sin duda necesario tener la capacidad de escucharla.

73 "All things of grace and beauty such that one holds them to one's heart have a common provenance in pain", ibídem, 54.

74 "Their birth in grief and ashes", ídem.

75 "There were times when he sat watching the boy sleep that he would begin to sob uncontrollably but it wasn't about death. He wasn't sure what it was about but he thought it was about beauty or about goodness", ibídem, 129. 


\section{Referencias bibliográficas}

Baudelaire, Charles. 1991. Oeuvres. París: Gallimard. Ed. de La Pléïade.

Baudelaire, Charles. 2000. Obras. Madrid: Espasa Calpe. Fundación Biblioteca de Literatura Universal.

McCarthy, Cormac. 2007. The Road. Nueva York: Vintage International.

McCarthy, Cormac. 2009. La carretera. Murillo Fort, Luis (trad.). Barcelona: Mondadori. 